\title{
Progiciel de Gestion Intégré : Entre l'artefact et l'objet social Un essai de lecture Structurationniste
}

\author{
Siham JABRAOUI \\ Professeur assistant Laboratoire de recherche ISO \\ Ecole Nationale de Commerce et de Gestion de Casablanca ENCG-C \\ Université Hassan II de Casablanca-Maroc
}

\begin{abstract}
The ERP integration project is to implement an external technological solution to solve internal problems of the company. The project to integrate such technology also becomes a project to restructure technology and organization according to the principles of the structurationist approach. An approach that we consider particularly suited to a conceptual reading of the problem of ERP integration, because it provides key concepts for the analysis of the organization, the technology that supports its management and the articulation between the two concepts through the integration project.
\end{abstract}

Keywords: Duality of technology, ERP, Integration, Organization, organizational change, Theory of structure.

Résumé : L'intégration d'un PGI consiste à mettre en place une solution technologique qui provient de l'extérieur pour résoudre des problèmes internes à l'entreprise. Le projet d'intégration de telle technologie devient par ailleurs un projet de restructuration de la technologie et de l'organisation selon les principes de l'approche structurationniste. Une approche que nous considérons particulièrement adaptée à une lecture conceptuelle de la problématique de l'intégration des PGI, puisqu'elle fournit des concepts clés pour l'analyse de l'organisation, de la technologie qui supporte sa gestion et de l'articulation des deux concepts à travers le projet d'intégration.

Mots clés: Changement organisationnel, Dualité de la technologie, Intégration, Organisation, PGI, Théorie de la structure,.

\section{Introduction}

La théorie de la structuration est un courant développé par le sociologue Giddens $(1976,1979,1987)$ [1]. Elle s'intéresse essentiellement à l'étude des rapports sociaux (Rojot, 2000) [2] notamment au niveau macro-social. La mobilisation des travaux de Giddens au niveau micro-social exigeait la reconsidération de la société par rapport à l'organisation. Giordano (1998) [3] considère que ce courant « offre pour les sciences de gestion, un cadre privilégié pour concevoir l'action organisée dans des termes souvent présentés comme exclusifs ou irréconciliables ». Cette appropriation a permis l'adoption de la théorie de la structure pour le développement des problématiques organisationnelles et technologiques telle que l'introduction de technologies nouvelles dans l'organisation. Ce qui explique l'intérêt que nous réservons à cette théorie pour encadrer la question de l'intégration des PGI. La mise en place de tels systèmes de par leur complexité ne doit pas négliger l'aspect humain d'échange qui est indispensable de même que la dimension organisationnelle.

Notre étude vise à intégrer l'ensemble des aspects organisationnel, technique et humain nécessaires à l'intégration des systèmes PGI dans un environnement multi-acteurs, complexe et dynamique. Elle met en avant l'importance des variables organisationnelles et technologique et surtout de leurs interactions dans la structuration et l'intégration de la technologie ( Deltour, Vaast 2000) [4]. Par ailleurs, notre objectif dans cet article est de montrer que la théorie de la structure peut être considérée comme un cadre théorique adéquat à l'étude du phénomène de l'intégration des systèmes PGI.

Nous allons tenter dans un premier temps, d'expliquer l'intérêt théorique de l'approche structurationniste pour les questions d'introduction, d'intégration et d'évolution de la technologie dans les organisations. Ensuite, nous allons faire le rapprochement entre cette théorie et notre question de recherche. Nous insisterons sur quelques concepts clés que nous jugeons applicables dans les projets d'intégration des systèmes PGI.

\section{L'approche Structurationniste : Un cadre conceptuel et des concepts clés pour l'analyse des organisations}

Nous cherchons à travers ce travail d'analyser la pertinence de l'approche structurationniste dans le cadrage théorique des problématiques issues du domaine des nouvelles technologies. Nous tacherons par la suite, à mettre l'accent sur les concepts clés de la structuration concernant de prés la question liée à l'intégration des PGI. 


\section{II-I La structuration: Un cadre conceptuel adéquat}

Les travaux de Giddens ont récemment inspiré beaucoup de chercheurs en sciences de gestion: Autissier et Wacheux (2000) [5], Giordano (1998) [6] et tant d'autres. La mobilisation de ce courant par les gestionnaires pour encadrer une variété de thèmes souligne l'importance de l'applicabilité potentielle des travaux de Giddens aux organisations. Ce qui différencie Giddens des autres auteurs c'est sa façon particulière d'exposer sa pensée en ce qui la différencie des autres pensées. La mobilisation de ses travaux exige, par ailleurs des chercheurs qui souhaitent l'exploiter, une redéfinition des concepts structurationnistes en fonction de ce qu'ils veulent en faire. Certains auteurs ont bien souligné cette "transferabilité" des concepts structurationnistes aux organisations. C'est le cas de Rojot (1998, 2000), Wacheux (2000), Cazal (2000), Autissier et Le Goff (2000) [7] ... etc. Parmi les premières recherches Françaises qui se sont basées sur la théorie de la structure pour une application dans un domaine particulier, nous citons les travaux de Bouchikhi (1990) [8] dédiés à l'étude du changement organisationnel et des technologies informatiques. Giordano (1998) elle, s'est concentrée sur la mobilisation de ce courant dans le domaine de la communication comme l'a fait Bailly (1998) dans le domaine de la GRH et Autissier (1998) dans le domaine du contrôle de gestion.

La théorie de la structure propose, en effet, une théorie du social. Toutefois, elle peut tout à fait correspondre aux attentes des chercheurs dans d'autres domaines d'application notamment en sciences de gestion (Rojot 2000) [9]. En effet, la littérature de ce courant nous a permis de relier les apports de la structuration à la compréhension des relations entre technologie et organisation. Le principal intérêt de la structuration pour la gestion, selon Autissier et Wacheux (2000) [10], est qu' «elle ne considère pas l'organisation comme une structure formelle, mais comme un lieu de structuration ». Le concept de la récursivité de l'action et de la structure tel qu'il est postulé par Giddens, nous permet d'envisager le système organisationnel comme un lieu de construction permanente d'actions et de projets. La structuration nous permet ainsi d'appréhender les événements dans une logique dynamique qui ne se limite pas à des relations causales. C'est cette approche interactive des relations technologie-organisation que nous avons souhaité mettre en évidence à l'aide de ce cadre conceptuel. Nous reviendrons par la suite sur des travaux de Giddens [11] qui ont été repris par d'autres auteurs dans l'étude des liens technologie-organisation. Auparavant, nous présenterons succinctement les principaux concepts de la structuration mobilisés dans l'analyse des organisations.

\section{II-II Des concepts clés pour l'analyse de l'organisation}

Notre objectif à ce niveau, consiste à faire l'état des concepts clés de la structuration utilisés dans l'étude de la technologie et qui correspondent au contexte mouvant de notre thème de recherche à savoir l'intégration des PGI. L'objectif principal de la théorie de la structure, tel que Giddens l'expose, est d'expliquer comment les structures se constituent dans l'action et inversement, comment l'action se constitue dans la structure. La théorie de la structuration repose donc sur la notion de « la dualité du structurel ». D'après Deltour et Vaast (2000) [12] « La structure et les propriétés institutionnelles des systèmes sociaux sont crées par l'action humaine et contribuent à former les futures actions humaines. Les structures sont produites et reproduites par interactions entre action et structure ». Cette notion a été retenue dans plusieurs travaux de recherche notamment ceux qui étudient les rapports entre la technologie, l'action humaine et la structure sociale ( DeScanctis et Poole 1994) [13].

Avant d'analyser ces rapports, il convient de préciser la notion de la structure à la différence de celle du système, telle qu'elle a été définie par Giddens. Sa théorie permet en effet, de mettre en avant l'organisation en tant que système d'activités caractérisé par deux propriétés essentielles : le caractère structuré (forme) et le caractère structurant (processus) de l'action. Giddens inscrit justement son approche de l'organisation dans le dépassement des dichotomies qui opposent ces deux caractères. "L'étude de la structuration des systèmes sociaux est celle des modes par lesquels ces systèmes, qui s'ancrent dans les activités d'acteurs compétents, situés dans le temps et dans l'espace et faisant usage des règles et des ressources dans une diversité de contextes d'action, sont produits et reproduits dans l'interaction de ces acteurs, et par elle» Giddens (1987). Elle offre ainsi un cadre intégrateur d'une approche de l'organisation en tant que structure ayant une forme concrète, et celle de l'organisation en tant que système d'activités. La dualité du concept de l'organisation permet de proposer une distinction pertinente entre «structure » et «système» sans pour autant les opposer. Une distinction qui peut être considérée en parallèle de celle proposée par Morin [14] entre «visage intériorisé » et « visage extériorisé » du système.

Le modèle structurationniste de Giddens repose ainsi sur l'analyse des interactions humaines produites et reproduites sur la base d'un ensemble de règles et pratiques organisées de façon récursive. L'organisation en tant que système apparaît à travers les activités individuelles et collectives des acteurs qui en font partie. Sa dimension structurelle apparaît au niveau du cadre de référence qu'elle propose pour l'accomplissement de ces actions et interactions dans des contextes de temps et d'espace particuliers et pour des finalités partagées. C'est un lieu de structuration des systèmes d'activités. Les structures organisent ces systèmes d'activités en même temps que ces derniers les organisent et leur donnent du sens. 
Giddens a spécifié le rôle des acteurs et leur action à travers la mobilisation de la notion du temps et de l'espace. L'organisation représente par ailleurs une variété d'interactions entre acteurs de différents niveaux d'organisation et dans des cadres spatio-temporels différents. Cette notion de différenciation a été mobilisée par Giddens pour introduire le concept de régionalisation quand il voulait désigner la différence des pratiques et des processus de structuration en fonction «des différentes régions » de l'organisation et de leurs relations avec leurs environnements.Cette question de régionalisation- différenciation nous a particulièrement interpelés, notamment son applicabilité à notre question de recherche. En effet, la mobilisation du concept de régionalisation du système organisationnel permettra sans doute de vérifier par la suite l'existence de différentes logiques dominantes de l'intégration des PGI adoptées par les différentes fonctions ou entités (régions) à l'occasion de leur implémentation dans le système organisationnel.

\section{III- La théorie de la structure et l'intégration des PGI}

III-I L'approche structurationniste de la technologie: Un cadre conceptuel approprié pour l'étude de l'intégration des PGI

Comme nous l'avons mentionné précédemment, plusieurs disciplines ont trouvé dans la théorie de la structure un cadre conceptuel pour l'analyse des situations de gestion. La question de l'intégration organisationnelle des PGI relevant essentiellement du domaine de la gestion. Elle peut aisément faire l'objet d'une application structurationniste. Notamment, après la mobilisation récente de la théorie de la structure dans des travaux d'analyse sur l'introduction et l'évolution des technologies d'information dans des organisations variées (Barley 1986, DeSanctis et Poole 1994, Groleau 2000, Orlikowski 1992, 1996, Tyre et Orlikowski 1994) [15]. En prenant appui sur la théorie de la structure pour encadrer la question de l'intégration organisationnelle des PGI, nous visons avant tout de situer notre raisonnement loin des modèles déterministes qui qualifient la relation organisation-technologie de causalité linéaire reposant souvent sur les caractéristiques de l'artefact. Pour mettre en évidence la nature interdépendante de ces relations, la théorie de la structure propose la notion de construction récursive entre organisation et technologie. Cette notion met en évidence le double caractère structurant/structuré de l'organisation comme de la technologie. Elle définit l'organisation et la technologie comme des processus qui se construisent mutuellement et de manière permanente. Dans le cadre de cette recherche, cette approche permet de rendre compte des différentes interactions opérées entre les variables technologiques et organisationnelles dans le cadre d'un projet d'intégration d'un PGI. Cette approche permet donc de qualifier l'intégration organisationnelle des PGI comme une co-construction permanente et partagée entre le PGI et l'organisation.

\section{III-II L'approche structurationniste du PGI : entre la technologie et l'objet social.}

Une analyse comparative de plusieurs études menées essentiellement dans le cadre de l'introduction ou la gestion des systèmes informatiques (Groleau, 2000) [16], démontre que l'application de la théorie de la structure se fait selon trois grandes perspectives : Une perspective qui considère la structuration des systèmes en tant que processus de structuration cognitive où les interactions humaines sont le moyen des acteurs pour changer les structures sociales (Newman \& Robey, 1992) [17]. Une perspective qui définit la technologie en s'appuyant sur l'aspect social des relations qu'elle entretient avec ses utilisateurs mais qui met plus l'accent sur une dimension volontariste que sur une dimension déterministe de la dualité technologique. Ainsi, les conséquences d'une technologie peuvent dépendre aussi bien de ses propriétés matérielles si elles sont aussi influentes que les contextes de son utilisation. Une perspective qui considère la structuration comme un processus d'appropriation qui permet l'adaptation des structures (DeSanctis et Poole, 1994) [18] qui sont en permanente production et reproduction dans le processus d'interaction via les technologies de l'information. Une perspective qui reconnaît le caractère matériel de la technologie (l'artefact) sans pour autant négliger le rôle des pratiques sociales dans la construction de cette technologie.

Une perspective qui suggère la dualité de la technologie comme point de départ à l'application de la théorie de la structure dans les problématiques d'introduction des technologies d'information. Ainsi, Orlikowski (1992) [19] considère la technologie comme le fruit d'une construction sociale résultant de l'action humaine et des propriétés structurelles du système organisationnel. Elle refuse toute considération exclusive, matérialiste et déterministe de la technologie comme une force objective ou le fruit d'une construction sociale. 


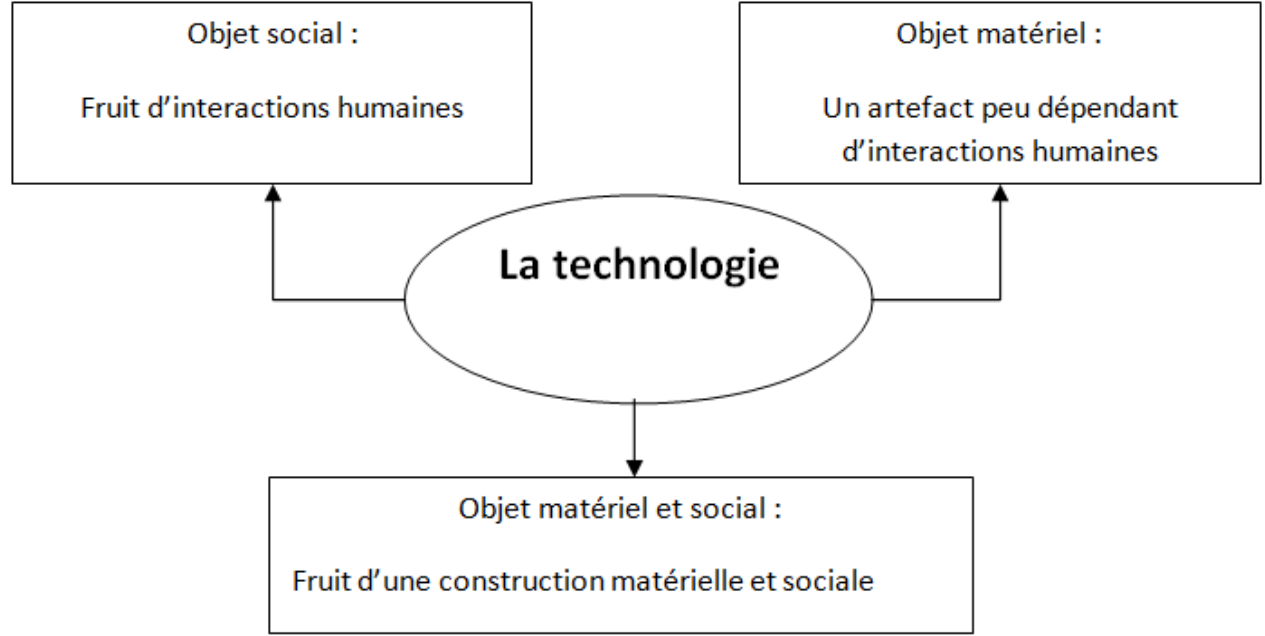

Figure 1 : Les différentes perceptions de la technologie

Pour appréhender la problématique d'intégration des PGI, notre recherche retient plutôt l'acception duale de la technologie. Une approche qui considère le PGI comme étant à la fois une nouvelle technologie (un objet matériel) et un projet organisationnel (un objet social). Selon cette perspective, le système PGI peut être construit par ses concepteurs à savoir les éditeurs, mais aussi par ses intégrateurs et ses usagers. Il peut donc être reconstruit au moment de l'intégration et de l'usage. Il se définit non seulement au travers l'artefact matériel (architecture applicative) mais aussi du point de vue des interprétations que l'en peut en faire (paramétrer ou développer à côté) et des évolutions que l'on peut y intégrer.

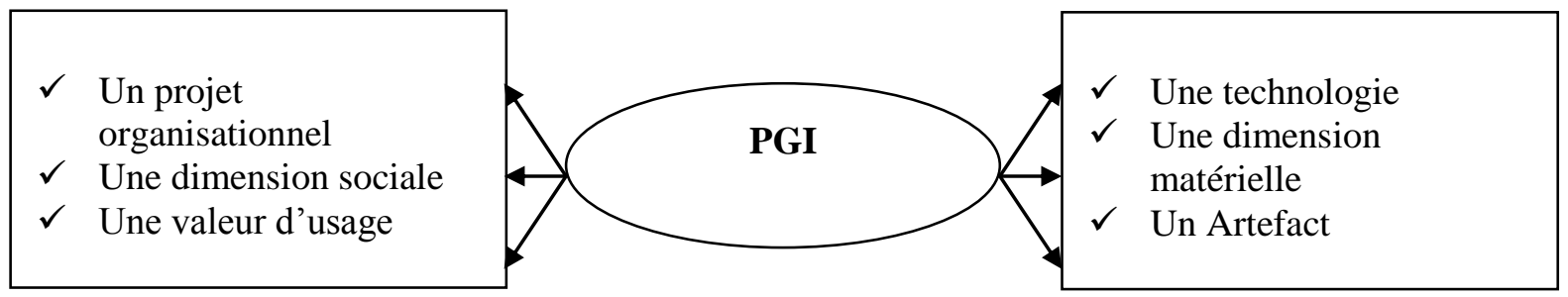

Figure 2 : les PGI : Une dimension duale

Les PGI requièrent leur dimension matérielle dans les caractéristiques techniques et technologiques du progiciel : c'est une application informatique Paramétrable (l'adaptation des besoins d'une entreprise donnée se fait par des paramétrage, choix des règles de gestion, d'options de traitement...), modulaire (un PGI est un ensemble de modules séparables correspondant chacun à un processus de gestion, et intégré (les différents modules échangent des informations et partagent une même base de données) (Reix, 2000) [20]. Un PGI en étant une technologie structurante, consiste à prendre une solution standard importée du dehors pour un problème de dedans. Il présente ainsi les caractéristiques d'un projet dans sa mise en place et sa gestion, mais diffère des autres projets par sa complexité, ses enjeux et ses effets. C'est un projet d'organisation d'une grande dimension sociale.

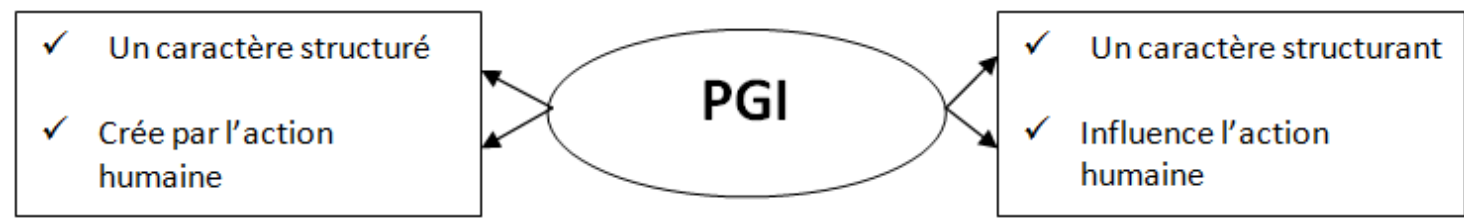

Figure 3 : PGI et action humaine

Le PGI étant à la base une donnée technologique, peut être considéré comme un construit social inachevé de l'action humaine. Ils demandent tout un processus pour être bien intégrés au niveau organisationnel. Cette intégration se fait essentiellement au niveau de la structure, de la stratégie et de la culture de l'entreprise en prenant en compte un certain nombre d'élément pour une meilleure préparation de l'entreprise et ses 
propriétés institutionnelles à une phase de changement inévitable quelle que soit sa nature : radicale ou incrémentale.

\section{Théorie De La Structuration : Une Théorie Du Changement}

L'application de la théorie de Giddens aux problématiques technologiques permet de mettre en évidence le processus de construction mutuelle et permanent entre technologie et organisation. L'approche structurationniste permet ainsi de concevoir la notion de changement organisationnel en termes d'interdépendances entre les variables technologiques et les variables organisationnelles. Ce cadre permet d'analyser la question d'intégration organisationnelle des PGI comme une co-construction partagée entre l'organisation et le système PGI. Les implications techniques et organisationnelles de cette construction ont fait l'objet de différents traitements selon l'approche de la technologie adoptée par les chercheurs. Ainsi, Barley rend compte de l'importance du contexte historique et des interactions sociales dans l'étude des influences des technologies sur les structures «si les technologies influencent les structures organisationnelles, leur influence dépend du contexte historique spécifique dans lequel elles sont enchâssées » (Barley, 1986) [21].

Pour caractériser les mêmes interactions technologie-organisation, Orlikowski (1992) [22] suggère de réconcilier les approches sociales et matérielles de la technologie dans un modèle structurationniste de la technologie « a structurational model of technology ». Pour élaborer ce modèle, Orlikowski s'appuie sur deux concepts de base : la dualité de la technologie et la flexibilité de ses caractéristiques matérielles et techniques « interpretive flexibility of technology ».

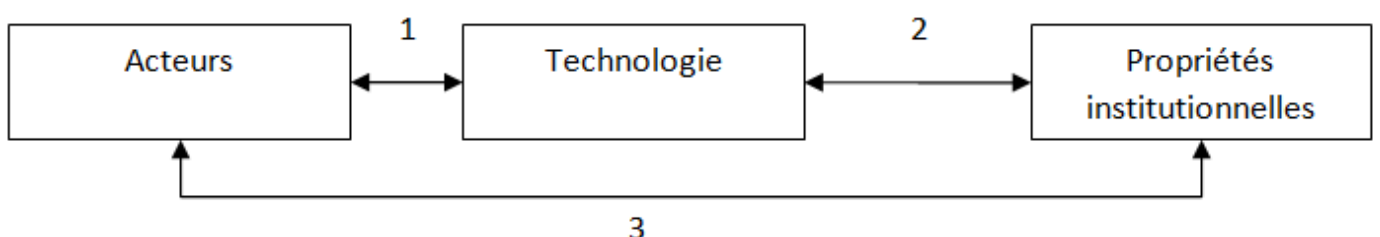

Figure 4 : Modèle structurationniste de la technologie (Orlikowski)

Ce modèle met en perspective le rôle des acteurs dans la co-construction de la technologie et de l'organisation. La construction d'une technologie comme le PGI ne se limite pas à sa conception chez son éditeur. Elle se construit également au moment de son usage (Tyre et Orlikowski, 1994) [23]. Ce qui qualifie ce modèle c'est la nature des relations qui lient ses composantes (acteurs, technologie et structure) qui interagissent de manière récursive.De Sanctis et Poole (1994) [24] propose un autre modèle pour qualifier les processus d'adaptation d'une nouvelle technologie " Adaptive Structuration Theory ». L'AST met en évidence l'émergence de nouvelles structures des interactions entre acteurs et technologies.

Les différents modèles et approches que nous venons de citer proposent une vision volontariste du changement organisationnel basée sur des relations récursives entre les variables technologiques et organisationnelles. Loin des modèles déterministes de la relation technologie-organisation, l'approche structurationniste des changements liés à l'intégration d'une technologie PGI, leur attribue un caractère imprévisible en raison de l'interaction non seulement des caractéristiques technologiques (PGI) et structurelles (organisation), mais également des jeux d'acteurs qui s'opèrent à l'occasion de ces projets.

\section{Conclusion}

L'idée fondamentale que nous inférons de la théorie de la structure par rapport à la question de l'intégration d'un PGI est que l'organisation est un lieu de structuration des systèmes d'activités. Les structures organisent ces systèmes d'activités, tout autant que ces derniers les organisent et leur donnent du sens et une finalité. Le PGI lui, est considéré par ailleurs comme un construit social et matériel à la fois selon le principe de la dualité de la technologie. Ce système socio-technique peut être à la fois structurant, structuré, proposant à l'organisation des possibilités de changement et de restructuration et imposant son caractère standard. Il peut être co-constructeur de la stratégie de l'entreprise au moment de sa définition et moyen de sa mise en place.

Il peut être à la fois résultat de son intégration à travers sa reconstruction et moyen de l'intégration de l'organisation. C'est à partir de ces idées que nous appliquerons la théorie de la structure à l'objet technologique PGI et plus particulièrement à son intégration au niveau organisationnel, en insistant sur le caractère dynamique de leur construction. Une co-construction partagée entre technologie et organisation à laquelle on associe un changement technico-organisationnel indissociable. 


\section{Bibliographie}

[1]. Giddens, A (1976), « New rules of sociological method: appositive critique of interpretative sociologies», London huichinson.Giddens, A (1979), «Central problems in social theory: action, structureand contradiction in social analysis », London: Macmillan.Giddens,A (1987), « La constitution de la société », Presse universitaire de France, Paris.

[2]. Rojot, J (2000), «La théorie de la structurationchez Anthony Giddens », dans Structuration et management des organisations, L'Harmattan, Paris, P 47-57.

[3]. Giordano, Y (1998), « La théorie de la structuration d'Anthony Giddens. Quels apports pour les sciences de gestion ? », Revue de Gestion des Ressources Humaines, N²6-27, mai-juin, 3-4.

[4]. Deltour, Vaast (2000), «Quand technologie et organisation construisent un réseau d'échanges professionnels : une étude de cas structurationniste ». Cinquième congrés de l'AIM, montpellier Novembre.

[5]. Autissier et Wacheux (2000), «Retours d'expérience d'actions de changement dans les entreprises » dans Structuration et management des organisations, L'Harmattan, 2000, p. 27-44.

[6]. Giordano,Y (1998), « La théorie de la structuration d'Anthony Giddens. Quels apports pour les sciences de gestion ? », Revue de Gestion des Ressources Humaines, N²6-27, mai-juin, 3-4.

[7]. Rojot, J (1998), «La théorie de la structuration », Revue de gestion des ressources humaines, n²6-27, mai-juin 1998, p. 5-19. Rojot, J (2000), «La théorie de la structurationchez Anthony Giddens », dans Structuration et management des organisations, L'Harmattan, Paris, 2000, p. 47-57.

[8]. Cazal, D (2000), «Réflexivité et organisations : critique et perspectives », in Structuration et Management des Organisations, L'Harmattan, 227-257.Wacheux, F (2000), « Le paradigme de la structuration sur l'analyse des situations de travail : effets en retour dans les processus de routinisation », in Structuration et Management des Organisations, L'Harmattan, 295 -320.

[9]. Romelaer, P (2000), « Rencontres et organisation », in Structuration et Management des Organisations, L'Harmattan, 59-97. Rojot, J et Wacheux, F (2000), «Le paradigme de l'interactionnisme symbolique sur l'analyse des situations organisationnelles : complémentarités et ambivalences avec la structuration », in Structuration et Management des Organisations, L'Harmattan, 259277.Autissier, D et Le Goff, J (2000), « Dualité du structurel et dynamique sectorielle : application à la distribution des composants électroniques » dans Structuration et management des organisations, L'Harmattan, p. 181-204.

[10]. Bouchikhi, H (1990), «Structuration des organisations : concepts constructivistes et études de cas », Paris, Economica

[11]. Rojot, J (2000), «La théorie de la structuration chez Anthony Giddens », dans Structuration et management des organisations, L'Harmattan, Paris, p. 47-57.

[12]. Autissier, D et Wacheux, F (2000), «Retours d'expérience d'actions de changement dans les entreprises » dans Structuration et management des organisations, L'Harmattan, p. 27-44.

[13]. Giddens, A (1987), « La constitution de la société », Presse universitaire de France, Paris.

[14]. Deltour, Vaast (2000), «Quand technologie et organisation construisent un réseau d'échanges professionnels : une étude de cas structurationniste ». Cinquième congrès de l'AIM, Montpellier Novembre.

[15]. DeScanctis et Poole (1994), « Capturing the complexity in advenced technology use, adaptative structuration theory ». Ed Science the institute of management. Vol 5 Organization science, p.121-147.

[16]. Morin E. (1984), « Sociologie », Paris : Fayard.

[17]. Barley, S.R (1986), «Technology as an Occasion for Structuring : Evidence from Observations of CT Scanners and the Social Order of Radiology Departments », Administrative Science Quarterly, N³1, 78-108.

De Sanctis G. and Poole M.S. (1994), «Capturing the Complexity in Advanced Technology Use : Adaptive Structuration Theory », Organization Science, Vol. 5, N4, 121-147.

Groleau, C (2000), « La théorie de la structuration appliquée aux organisations : le cas des études sur la technologie », in Structuration et Management des Organisations, L'Harmattan, 155-179.

Orlikowski, W.J. (1992), « The Duality of Technology : Rethinking the Concept of Technology in Organizations », Organization Science, Vol. 3, N³, August, 398-427.

Orlikowski, W.J. (1996), «Improvising Organizational Transformation over Time : A Situated Change Perspective », Information Systems Research, 7, 63-92.

Tyre, M.J. and Orlikowski, W.J. (1994), «Windows of Opportunity : Temporal Patterns of Technological Adaptation in Organizations », Organization Science, Vol. 5, $\mathrm{N}^{\circ} 1,98-117$

[18]. Groleau, C (2000), «La théorie de la structuration appliquée aux organisations : le cas des études sur la technologie », in Structuration et Management des Organisations, L'Harmattan, 155-179.

[19]. Newman, M and Robey, D (1992), « A Social Process Model of User-Analyst Relationships », MIS Quarterly.

[20]. DeSanctis, G. and. Poole M.S (1994), « Capturing the complexity in advanced technology use: Adaptive structuration theory », Organization Science.

[21]. Orlikowski, W.J. (1992), « The Duality of Technology : Rethinking the Concept of Technology in Organizations », Organization Science, Vol. 3, N³, August, 398-427.

[22]. Reix, R (2000), «Systèmes d'information et management des organisations », Vuibert 3ème édition.

[23]. Barley, S.R. (1986), « Technology as an Occasion for Structuring : Evidence from Observations of CT Scanners and the Social Order of Radiology Departments », Administrative Science Quarterly, N³1, 78-108.

[24]. Orlikowski, W.J. (1992), « The Duality of Technology : Rethinking the Concept of Technology in Organizations », Organization Science, Vol. 3, N³, August, 398-427.

[25]. Tyre, M.J. and Orlikowski, W.J. (1994), «Windows of Opportunity: Temporal Patterns of Technological Adaptation in Organizations », Organization Science, Vol. 5, N¹, 98-117

[26]. De Sanctis, G. et Poole, M.S. (1994), « Capturing the Complexity in Advanced Technology Use : Adaptive Structuration Theory », Organization Science, Vol. 5, N4, 121-147. 